\title{
Development of agricultural bionic mechanisms: Investigation of the effects of joint angle and pressure on the stability of goats moving on sloping lands
}

\author{
Fu Zhang ${ }^{1,2 *}$, Limin Zheng ${ }^{1}$, Wei Wang ${ }^{1}$, Yafei Wang ${ }^{1}$, Jiajia Wang ${ }^{1}$ \\ (1. College of Equipment Agricultural Engineering, Henan University of Science and Technology, Luoyang 471003, China; \\ 2. Collaborative Innovation Center of Machinery Equipment Advanced Manufacturing of Henan Province, Luoyang 471003, China)
}

\begin{abstract}
The use of small-scale agricultural machinery becomes prevalent as it provides the stability of agricultural machinery research ideas. To lay the theoretical foundation for the research and design of agriculture walking mechanism, the Phantom Camera Control software was used to measure the anterior and posterior joint angle of a goat walking on different slopes. Foot pressure was measured by the film pressure sensor. The result of joint motion sequence, range of motion, and change of range showed that the fitting degree of the measured value was accurate. As the goat walking speed increased, the level of the hind limb angle changed to ensure itself stability. When the goat is walking, the forelimbs bear more weight than the hind limbs due to the different static and physiological structures of the front and rear legs. The key parameters of gait on different slopes were analyzed. The curve of angle change of legs was measured and analyzed when the goat is walking in slope. The results showed that, with the increase of the slope gradient, the anterior hip angle ranges from $83.3^{\circ}$ to $117.1^{\circ}$, the posterior hip angle ranges at $120.3^{\circ}-173.1^{\circ}$, the left knee angle ranges from $91.3^{\circ}$ to $170.1^{\circ}$, the right knee angle is roughly the same as its range of variation. When the slope increased, the pressure change of left hind foot was consistent with that of the right anterior foot, and the pressure change of right hind foot was consistent with that of the left anterior foot. This demonstrated the theory of diagonal gait. Meanwhile, with the change of the slope, the plantar pressure of the limbs changes periodically. The research results verified the rationality of the four-legged bionic mechanism under various parameters, which can provide a theoretical basis for the design of agricultural walking mechanism to adapt different slopes in the hilly and mountainous areas. Keywords: bionic mechanisms, goat, slope, joint angle, pressure, stability DOI: $10.25165 /$ j.ijabe.20181103.3633
\end{abstract}

Citation: Zhang F, Zheng L M, Wang W, Wang Y F, Wang J J. Development of agricultural bionic mechanisms: Investigation of the effects of joint angle and pressure on the stability of goats moving on sloping lands. Int $\mathrm{J}$ Agric \& Biol Eng, 2018; 11(3): 35-41.

\section{Introduction}

Bionics is a comprehensive interdisciplinary subject combined with life science, mathematics and engineering technology. Study on the entire structure, movement mode, control strategy of animals, and the moving mechanism of mountains is important. Chen et al. ${ }^{[1]}$ first established the model of the bionic locust robot, and kinematic analysis was performed to determine the relationship between landing positions and the legs attitudes. Then the locust's landing buffering processes were described. And legs attitudes determination method was proposed based on the landing buffering performance. Beijing University of Aeronautics and Astronautics cooperated with the University of Hamburg in Germany to develop a multi-joint micro-robot imitating caterpillars that can crawl on a

\section{Received date: $2017-12-05$ Accepted date: $2018-02-10$}

Biographies: Limin Zheng, Master candidate, research interest: intelligent agricultural equipmentand technology, Email: 870807613@qq.com; Wei Wang, Master candidate, research interest: intelligent agricultural equipment and technology, Email: 1091131762@qq.com; Yafei Wang, Master candidate, research interest: intelligent agricultural equipment and technology. Email: 946584218@qq.com; Jiajia Wang: PhD, Associate Professor, research interest: biomechanics and bionic, Email: johnnyjiajia@163.com.

*Corresponding author: Fu Zhang, PhD, Professor, Assistant Dean, research interests: biomimetic technology and the intelligent agricultural equipment. College of Equipment Agricultural Engineering, Henan University of Science and Technology, No.263, Kaiyuan Road, Luoyang 471003, China. Tel: +8613592065683, Email: zhangfu30@126.com. smooth surface ${ }^{[2]}$. In order to solve the problem of walking stability of small agricultural machinery, to provide the stability of agricultural machinery research ideas, a goat was selected as research object in this article.

At present, high-speed photography was widely used in the movement analysis of living organisms ${ }^{[3-6]}$. Marghitu et al.$^{[7]}$ obtained the kinematic parameters of the hind limb joints in canine trochanter with a camera with a motion analysis system, and analyzed the dynamic stability of the dogs using point-mapping techniques and nonlinear dynamic theory. With the appearance of the force-measuring plate, forces and joint moments can be measured during the analysis. Ground-based reaction forces and the measurement of propulsive forces were used to evaluate the walking gait of animals ${ }^{[8]}$. Budsberg et al. ${ }^{[9]}$ used force plates to acquire kinetic data and based on the vertical reaction force and the morphological correlation between the research force and body parameters. Tashman et al. ${ }^{[10]}$ studied hounds lacking anterior cruciate ligament and measured the kinematic data of the cruciate ligaments using the RSA system. The kinematic data of slow cruciate hounds were measured and the results were compared with those of joints and ligaments Injury-related factors, the establishment of a ligament injury and the relationship between the kinematics. Tokuda et al. ${ }^{[1]}$ proposed a method to estimate fragile footholds using the foot's center of force and pressure changes. Although their quadruped robot could detect when a foothold was collapsing, they did not propose how to make the robot walk on fragile terrain without stumbling. Xuan et al. ${ }^{[12]}$ 
taken the four-legged walking robot as the research object, analyzed the leg structure of the platform and deduced the calculating formula of the leg joint rotation angle. The main influencing factors of the stable gait were discussed and the steps from starting to walking steadily State adjustment mode, planning a steady cycle of gait.

Piórek et al. ${ }^{[13]}$ described an example of application of a nonlinear time series. The research aimed at assessment of the influence of both walking speed and ground slope on the resilience of gait control to infinitesimally small perturbations that occur naturally during walking. Liu et al. ${ }^{[14]}$ developed a staged evolutionary algorithm to tune system parameters and walking performance was improved. The developed control strategy was tested using a humanoid robot on irregular terrains. What's more, an automated computational system of the stable workspace was developed and an example was given to illustrate the whole process in detail. The theory and analysis procedures were also verified by simulation of the robot and its actual grabbing of an object ${ }^{[15]}$. Deng et al. ${ }^{[16]}$ proposed a new control strategy based on rolling gait and trajectory planning, which enables hexapod robot to walk through dynamic environments. The core point of this control strategy was to constantly change gait and trajectory according to different environments and tasks as well as stability state of robot. A control simulation platform was established to obtain the optimal controller parameters, and all the proposed methods were finally verified through simulations and experiments ${ }^{[17,18]}$. Some researchers $^{[19-21]}$ studied and implemented a real-time robust balance control for a humanoid robot under environment disturbance, the robot with robust control can resist an external thrust, stand on a two-axis inclinable platform, or walk on a see-saw successfully. Agheli et al. ${ }^{[22]}$ presented the Foot Force Stability Margin which was applicable to all types of multi-legged and multi-wheeled robots. A modified extension of the Foot Force Stability Margin was provided and compared to a widely used stability margin through a numerical simulation. In a study of sport lambs, in which motion gait and acquired motion data were observed, kinetic analysis of rock sheep provided the theoretical basis for a motion analysis system ${ }^{[23-25]}$. Xu et al. ${ }^{[26,27]}$ built a three-dimensional gait test system and the gait of blue sheep was tested on it. A moving image was processed by using the Video for Windows method to obtain the position and motion parameters of the limbs and provide the theoretical basis for the development of bionic rock sheep robots.

The current problem was that there are few researches on the application of bionic institutions in agriculture, and the specific measurement of bionic prototypes is not enough. To study the fitting of the main joint angles, the changing of foot pressure in a quadruped walking on slopes and to analyze their influence on stability. Different gradient goats walking curves were compared by using software measurement and data point drawing method in this paper. The movement of the quadruped while walking on a slope should be more fully understood, and the problem of small machines moving on irregular ground should be better addressed. On the basis of analysis of the foot structure and movement of a goat, according to the bionic principle, the movement mechanism of a goat walking on a slope can provide a theoretical basis for in-depth study and design of a slope walking mechanism ${ }^{[28-30]}$. As goats often walk on rugged ground relative to other quadrupeds, their foot surfaces have evolved to adapt to different surface geometries, with less resistance to movement, greater ability to pass and more efficient walking on the slope. Therefore, the walking of goat was measured and analyzed in this article.

\section{Test conditions and methods}

A six-month-old male goat in good condition and with a normal figure was analyzed in the test, as shown in Figure 1. The main body features are shown in Table 1. The experiment was performed in Luoyang Tractor Research Institute in, Luoyang, Henan Province.

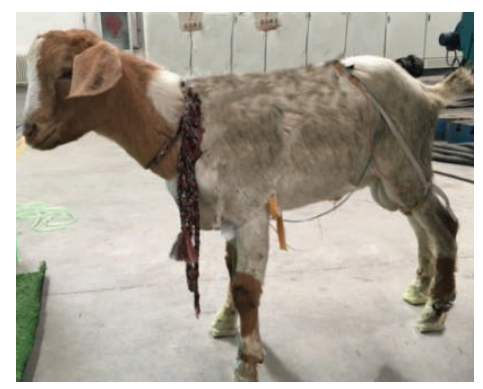

Figure 1 Photo of the goat

Table 1 Geometrical configurations of the goat

\begin{tabular}{lc}
\hline \multicolumn{1}{c}{ Parameters } & Value \\
\hline Weight, $m / \mathrm{kg}$ & $18.30 \pm 0.01$ \\
Body length, $l_{l} / \mathrm{m}$ & $0.86 \pm 0.01$ \\
Body height, $l_{h} / \mathrm{m}$ & $0.55 \pm 0.01$ \\
Front leg length, $l_{f} / \mathrm{m}$ & $0.19 \pm 0.01$ \\
Front thigh length, $L_{f} / \mathrm{m}$ & $0.20 \pm 0.01$ \\
Hind leg Length, $l_{b} / \mathrm{m}$ & $0.20 \pm 0.01$ \\
Hind thigh length, $L_{b} / \mathrm{m}$ & $0.22 \pm 0.01$ \\
\hline
\end{tabular}

The goat body characteristics were measured, and the measurements of the goat posture are shown in Table 1. Body length refers to the distance from the front and back of the hip on the same side of the goat, the height of the body refers to the distance from the ground to the goat hip.

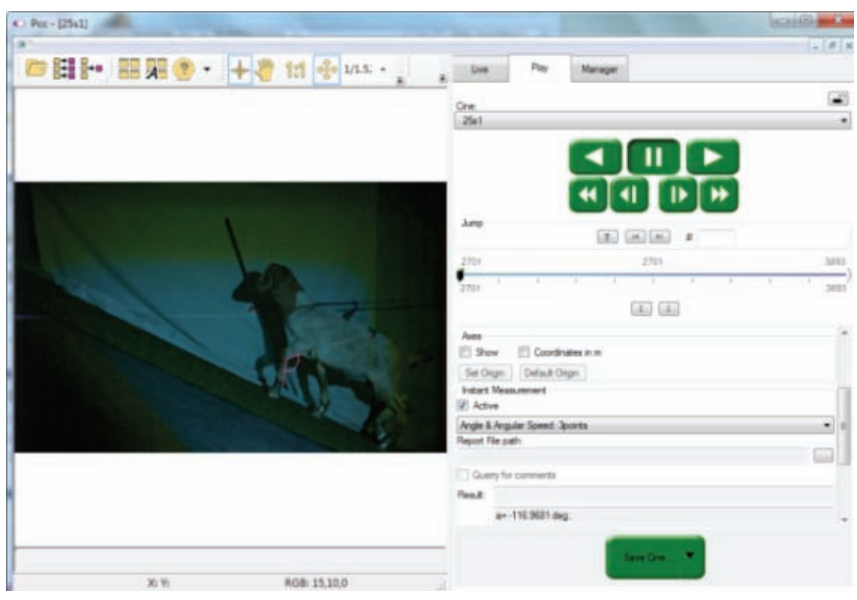

Figure 2 Parameter measurement

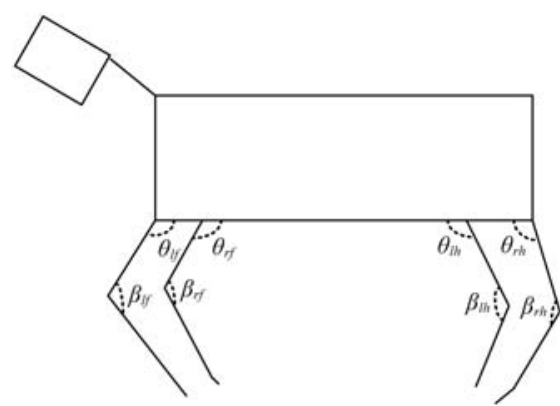

Figure 3 Schematic drawing of goat 
Figure 2 shows the "quick measurement" function of the Programmable Computer Controller software, in which the joint angles of goats at $0^{\circ}, 10^{\circ}, 20^{\circ}$, and $30^{\circ}$ slopes were measured. Figure 3 shows the goat limbs, including some information, as shown in Table 2.

Table 2 Body angle of the goat

\begin{tabular}{lc}
\hline \multicolumn{1}{c}{ Angle type } & Symbol name \\
\hline Left anterior hip angle & $\theta_{l f}$ \\
Left posterior hip angle & $\theta_{l b}$ \\
Right anterior hip angle & $\theta_{r f}$ \\
Right posterior hip angle & $\theta_{r b}$ \\
Left anterior knee angle & $\beta_{l f}$ \\
Left posterior knee angle & $\beta_{l b}$ \\
Right anterior knee angle & $\beta_{r f}$ \\
Right posterior knee angle & $\beta_{r b}$ \\
\hline
\end{tabular}

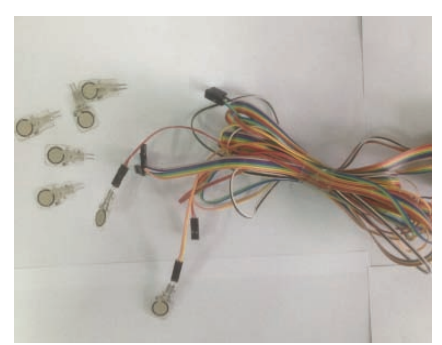

a. FlexiForce film pressure sensor A301

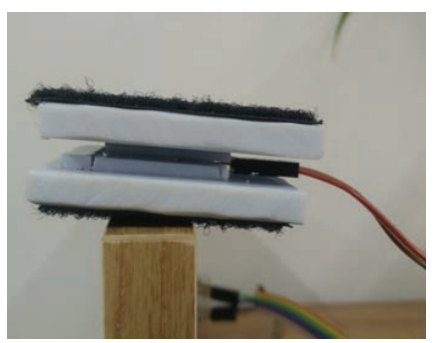

b. Sensor fixture

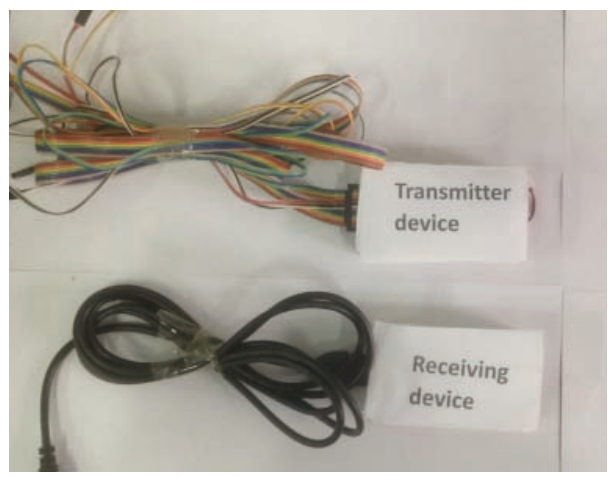

c. Wireless transmission

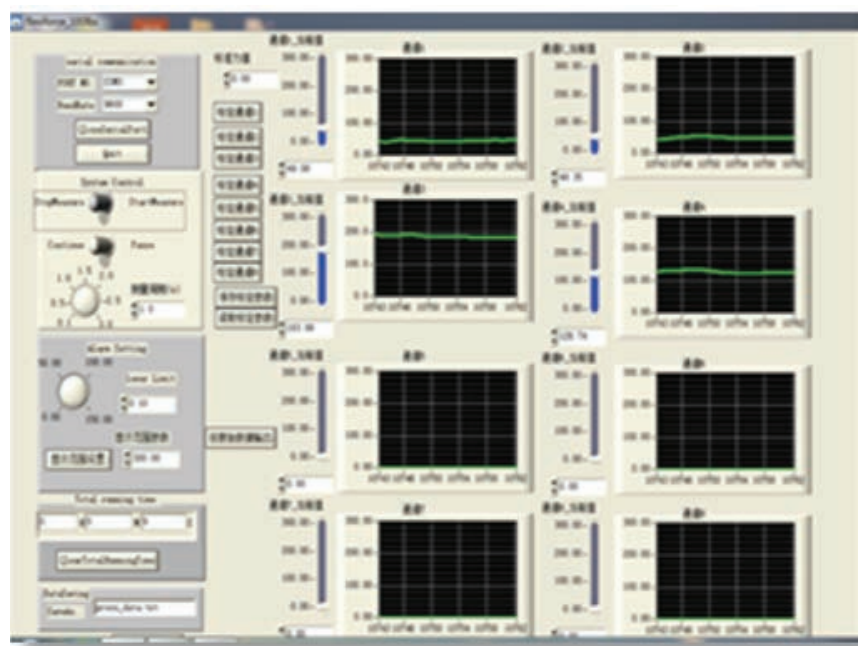

d. FlexiForce PC software

Figure 4 Wireless pressure measurement system

The wireless pressure measurement system is composed of a FlexiForce film pressure sensor, sensor fixture, wireless transmission module, and FlexiForce PC software constitute the wireless pressure measurement system. The pressure sensor is connected to the wireless transmitter by colored wires, and the wireless receiver is connected to the host computer software through the USB interface. The pressure of the goat foot is transmitted to the sensor through the sensor fixture. The sensor transmits the measured signal to the wireless transmitter. The wireless receiver stores the collected information on the computer and displays the real-time curve in the host computer software. The pressure sensor is attached to the center of the goat's foot by means of a sensor fixture, and the plantar pressure applied by the goat while walking on the different slopes is measured by the wireless pressure measurement system. The Origin software was used to analyze the pressure data and draw the pressure-time curve.

\section{Test data analysis}

\subsection{Analysis of the angle of goat joint}

The goat in the test is shown in Figure 5. The fitting curves of the angle between the hip joint and the knee angle of the goat walking on each slope are shown in Figures 6 and 7.

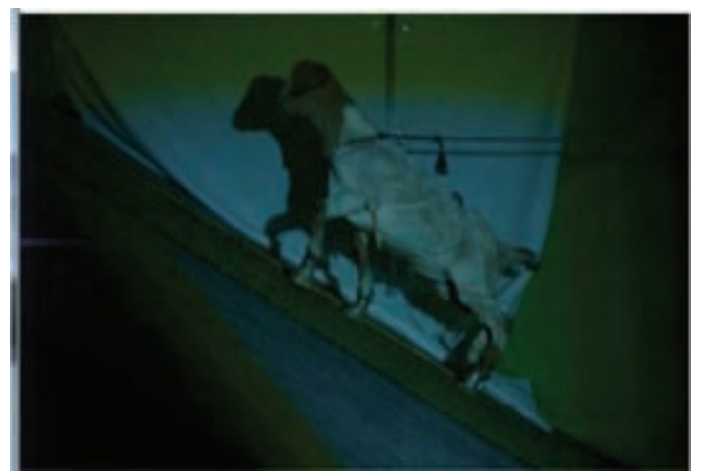

Figure 5 Goat walking during the test

The goats were repeatedly tested along the ascending direction at multiple slopes, and the images were taken from the time when the left front leg was lifted until a complete cycle was reached. The angle values of multiple tests were measured and the points with good regularity were comparatively analyzed. Figure 6a indicates that during level locomotion, the angle of $\theta_{l f}$, which gradually increases from $83.3^{\circ}$ to $117.1^{\circ}$ in 0 to $0.12 \mathrm{~s}$, reaches the maximum with stretched legs in $0.12 \mathrm{~s}$. The angle gradually reduces from $117.1^{\circ}$ to $48.3^{\circ}$, reaching the minimum in $0.33 \mathrm{~s}$, which is in the legged state. Moreover, the angle remaines unchanged at $0.43 \mathrm{~s}$ to $0.48 \mathrm{~s}$ due to the delayed action of the diagonal gait. When the slope increases to $10^{\circ}$ as shown in Figure $6 \mathrm{~b}$, the angle increases as the time period increases, reaching a maximum of $123.2^{\circ}$. When the slope increases to $20^{\circ}$ in $0.62 \mathrm{~s}$, the angle reaches the minimum value of $60.0^{\circ}$ in $0.11 \mathrm{~s}$. In the increasing state, the maximum value of $123.2^{\circ}$ reaches in $0.62 \mathrm{~s}$ as shown in Figure 6c. When the slope increases to $30^{\circ}$ as shown in Figure $6 \mathrm{~d}$, the angle first increases and then fell at $0.22 \mathrm{~s}$, reaching a maximum of $133.2^{\circ}$. The minimum value of $59.7^{\circ}$ reached in $0.68 \mathrm{~s}$.

Figure 6a shows that the angle of $\theta_{l b}$ gradually decreases and changes slowly within the period of $0-0.24 \mathrm{~s}$. However, the angle of the diagonal gait is almost unchanged within the period of $0.05-0.14 \mathrm{~s}$. The angle gradually increases from $134.4^{\circ}$ between $0.25-0.46 \mathrm{~s}$, and the goat is in the state of leg lift. After $0.46 \mathrm{~s}$, the angle gradually decreases in the leg lift state. When the slope increases to $10^{\circ}$ and $20^{\circ}$ as shown in Figures $6 \mathrm{~b}$ and $6 \mathrm{c}$, the angle oscillation amplitude decreases minimally, indicating that the right hind leg of the goat is in a state of adjusting gait. $\theta_{r f}$ and $\theta_{l f}$ indicate an opposite condition. $\theta_{r b}$ and $\theta_{l b}$ change in the opposite direction. At a $0^{\circ}$ slope as shown in Figure $6 \mathrm{a}$, minimum and 
maximum differences of time are observed due to the delay of diagonal gait. The same situation applies to $\theta_{r b}$ and $\theta_{l b}$.

In the abovementioned gradient conditions of goat joint curve,

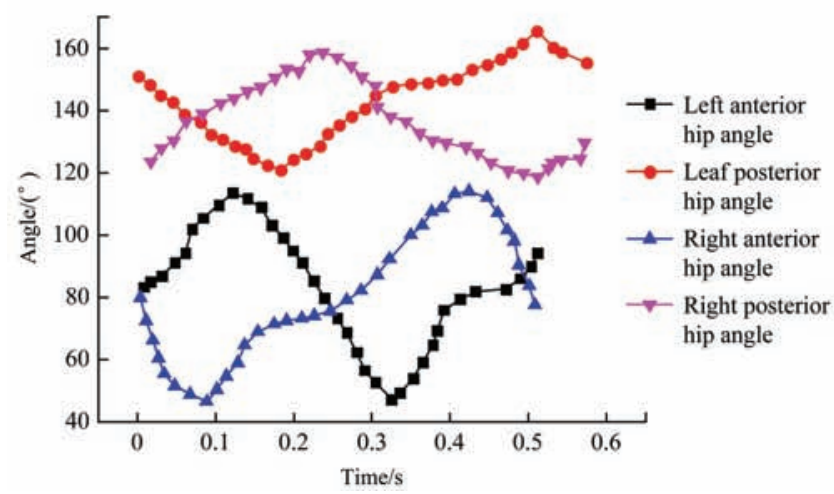

a. $0^{\circ}$ slope

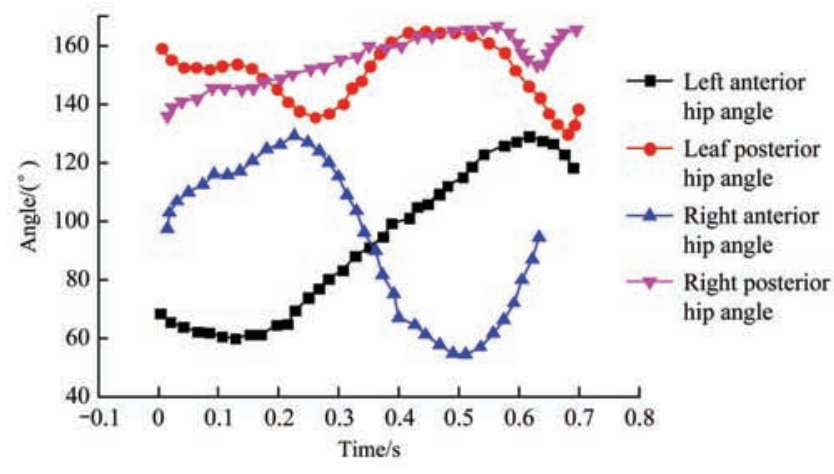

c. $20^{\circ}$ slope for the knee angle, the left angle of the left foreleg and the right front leg is opposite to the joint angle curve of the left hind leg and the right hind leg.

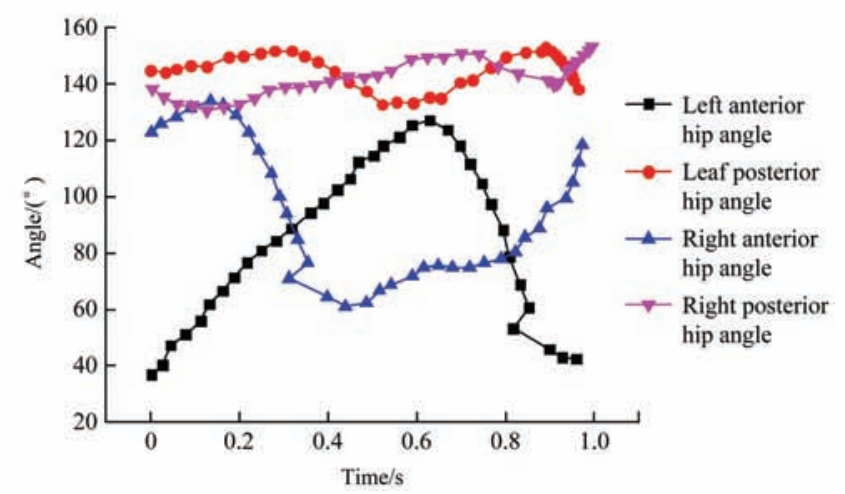

b. $10^{\circ}$ slope

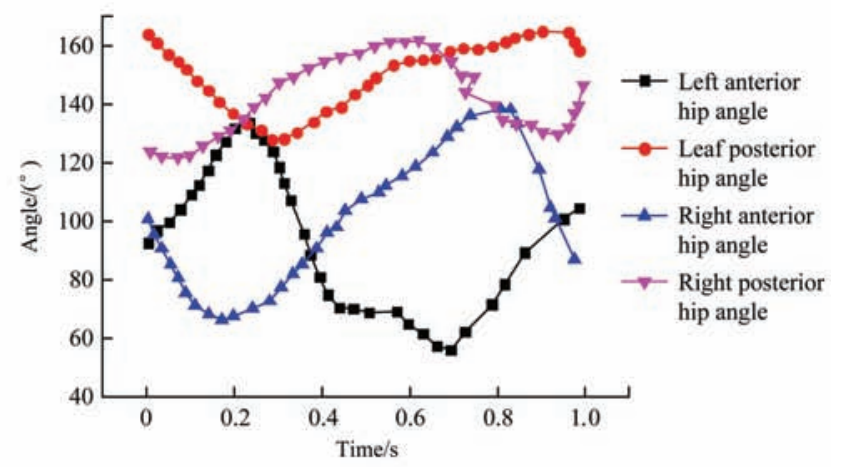

d. $30^{\circ}$ slope

Figure 6 Hip angle change curve of goat limbs on different slope

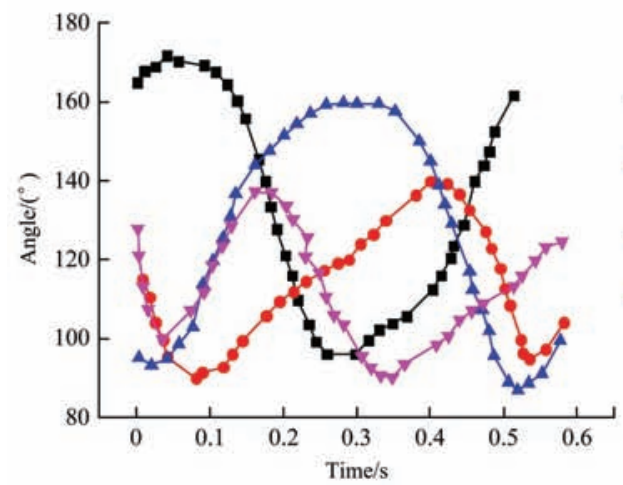

a. $0^{\circ}$ slope

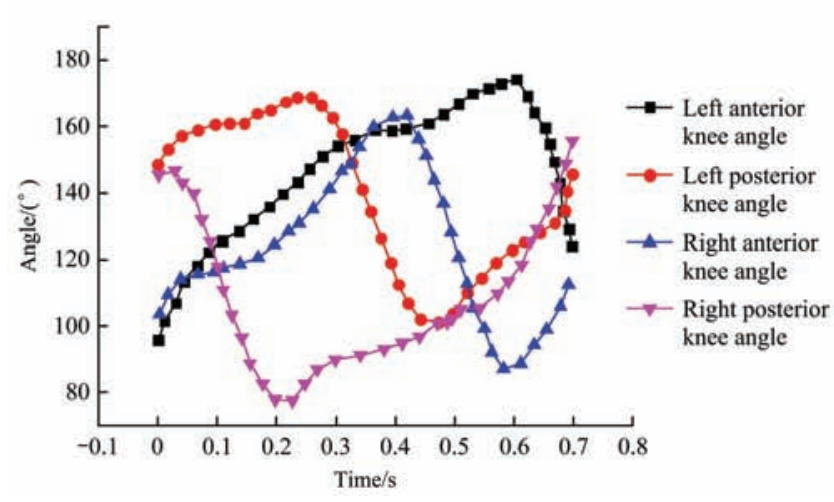

c. $20^{\circ}$ slope

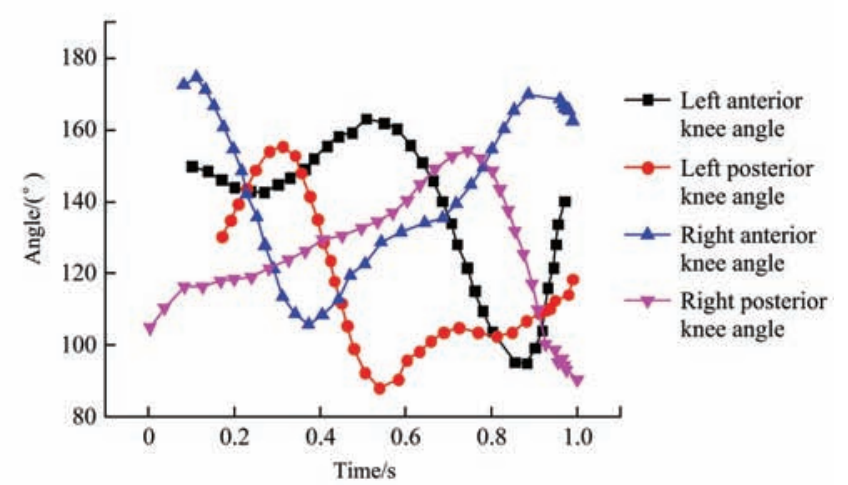

b. $10^{\circ}$ slope

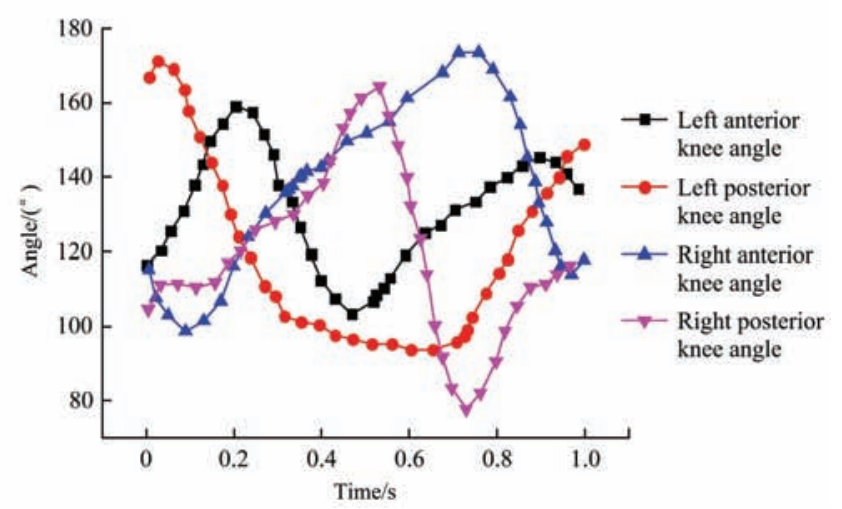

d. $30^{\circ}$ slope

Figure 7 Curves of goat knee angle changes with time on various slopes

In this study, the test was repeated a number of times, and a continuous and full cycle of walking data was selected. Figure 7a shows that $\beta_{l f}$ is unchanged during level locomotion at a maximum of $170.1^{\circ}$ between $0.03-0.09 \mathrm{~s}$ due to the left front leg and the 
cushioning effect of the ground. The angle quickly decreases to a minimum of $91.3^{\circ}$ at $0.09-0.26 \mathrm{~s}$, which is the leg lift state, and then gradually increases with time. When the slope increases to $10^{\circ}$ as shown in Figure $7 \mathrm{~b}$, the angle changes slowly between $0.06-0.52 \mathrm{~s}$ and reaches a maximum of $162.9^{\circ}$ at $0.52 \mathrm{~s}$, and then the angle decreases rapidly to a minimum of $91.2^{\circ}$. When the slope increases to $20^{\circ}$ as shown in Figure $7 \mathrm{c}$, the angle continuously increases until $0.59 \mathrm{~s}$, when it reaches a maximum of 169. $7^{\circ}$. When the slope increases to $30^{\circ}$ as shown in Figure $7 \mathrm{~d}$, the angle increases and reaches a maximum of $158.2^{\circ}$ at $0.17 \mathrm{~s}$ and a minimum of $108.4^{\circ}$ at $0.43 \mathrm{~s}$.

$\beta_{r b}$ and $\beta_{r f}$ change in opposite directions, thereby $\beta_{r b}$ reached a maximum value of $161.5^{\circ}$ at $0.31 \mathrm{~s}$ before the leg in the state. It then switches to the leg lift state when the angle is gradually reduced. When the slope increases to $10^{\circ}$ or $20^{\circ}$ as shown in Figures $7 \mathrm{~b}$ and $7 \mathrm{c}$, the same trend happened. $\quad \beta_{r f}$ and $\beta_{r b}$ change roughly before $0.15 \mathrm{~s}$ during level locomotion and then follow the opposite trend. $\beta_{r f}$ reaches the maximum and $\beta_{r b}$ reaches the minimum within the time difference of $0.04 \mathrm{~s}$ due to the diagonal gait delay. The trends of $\beta_{r f}$ and $\beta_{r b}$ are initially roughly identical and then become opposites on the $10^{\circ}$ slope as shown in Figure $7 \mathrm{~b}$. On the $20^{\circ}$ slope as shown in Figure $7 \mathrm{c}, \beta_{r f}$ reaches the maximum and $\beta_{r b}$ reaches the minimum value of time difference of 0.02 and $0.46 \mathrm{~s}$ before their change trends follow opposite directions and then return to the same state. On the $30^{\circ}$ slope as shown in Figure $7 \mathrm{~d}$, the trends of $\beta_{r f}$ and $\beta_{r b}$ change roughly before $0.47 \mathrm{~s}$ and then

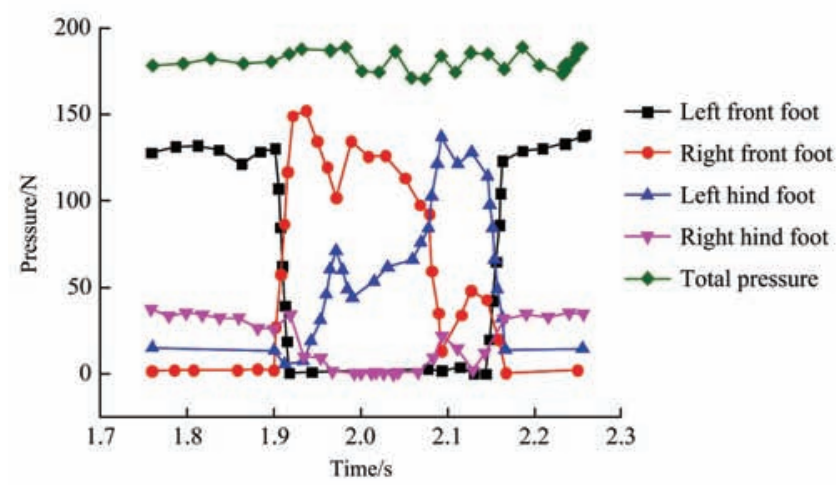

a. $0^{\circ}$ slope

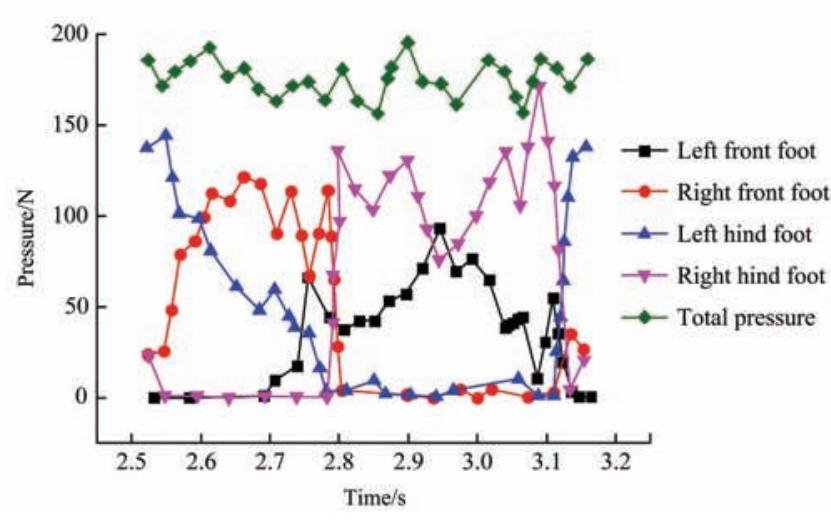

c. $20^{\circ}$ slope go in opposite directions.

In the abovementioned gradient conditions of goat joint curve, for the hip angle, hind legs are greater than the front legs. Moreover, the difference between the maximum angle and the minimum angle increases as the slope increases, thereby providing a research basis for imitating the walking trajectory of goats.

\subsection{Analysis of goat foot pressure}

A wireless pressure measurement system that consists of four components is established to study the characteristics of goat foot-ground contact and measure the pressure of the feet of the goat in this test.

The curves of the plantar pressure on the different slopes are shown in Figure 8. The left forefoot, right forefoot, left hind foot, and right hind foot pressures were expressed as $N_{1}, N_{2}, N_{3}$, $N_{4}$, respectively. On the $0^{\circ}$ slope, the left hind legs are in contact with the ground when $N_{1}$ reaches the maximum pressure of $140 \mathrm{~Pa}$, and the minimum and maximum pressures have a significant time difference due to the buffering between the feet and the ground. In addition, force alternates among the limbs. The forelimbs support more weight than the hind limbs because the relatively heavy center of the goat's body needs ample forelimb support. When the slope increases to $10^{\circ}$ or $20^{\circ}$, the buffer effect in the left front leg and right hind leg is substantial because of the long contact time with the ground. The buffering effect is significant for the left hind leg and the right front leg on the $30^{\circ}$ slope.

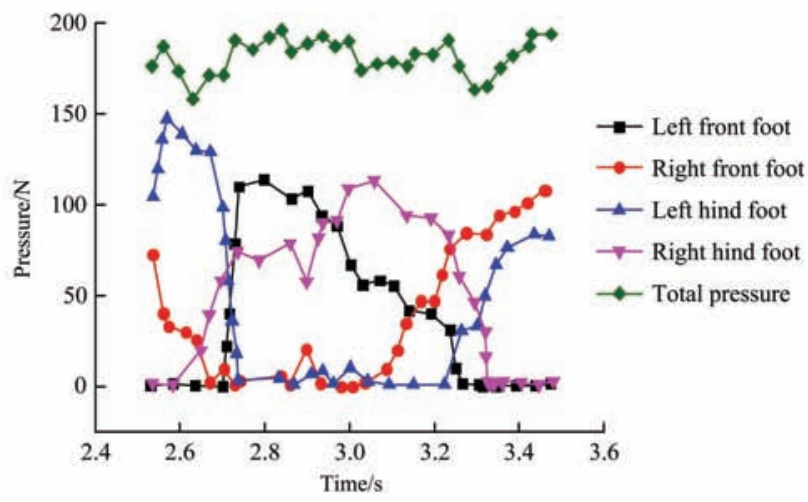

b. $10^{\circ}$ slope

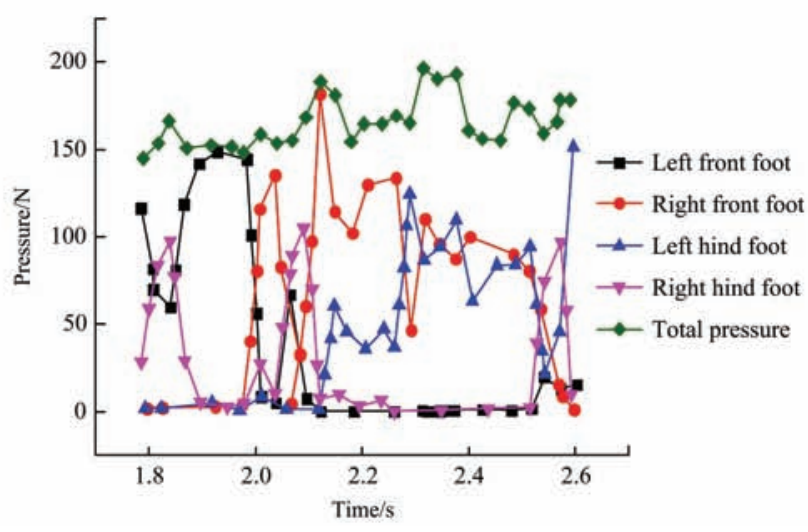

d. $30^{\circ}$ slope

Figure 8 Curves of plantar pressure

The hind legs of the goat push the ground to move the center of gravity toward the front while walking. The fore limbs are also subjected to this pressure. The front legs need to move the center of gravity forward to bear the weight of the goat's body. However, the front legs cannot fully support the center of gravity.
Therefore, the other legs also need to balance. The changes in $N_{1}$ and $N_{4}$ are consistent, and the change trends of $N_{2}$ and $N_{3}$ are consistent, thereby indicating that the goat had a diagonal trot gait on the four slopes. The trend of the total pressure of the limbs fluctuates around the variables $m g \cos \alpha$ with changes in 
the slope.

\section{Results and discussion}

A slope test scheme was designed and established to study the walking parameters of a goat on various slopes. The scheme comprises a system that measures the pressure applied by the goat through its feet, an automatic slope adjustment system that provides test slopes with $0^{\circ}, 10^{\circ}, 20^{\circ}$, and $30^{\circ}$ angles, and a high-speed camera system that records images of the goat's movement.

(1) When the slope gradient increases, the anterior hip angle changes from $83.3^{\circ}$ to $117.1^{\circ}$, and the posterior hip angle varies from $120^{\circ}$ to $173.1^{\circ}$. Thus, $\theta_{r f}$ and $\theta_{l f}$ are opposite, as well as $\theta_{r b}$ and $\theta_{l b}$. The knee joint angle ranges from $91.3^{\circ}$ to $170.1^{\circ}$, and $\beta_{l b}$ and $\beta_{l f}$ change in opposite directions. Compared with the forelimb, the range of angle changes of each joint was increased in the hindlimb, and the change in the angle of the joint was related to the role played by the anterior and posterior limbs.

(2) As the slope increases, the left forefoot, right hind foot, the left hind foot and right forefoot alternately get in contact with the ground and produce the buffer effect. $N_{1}$ coincides with the change in $N_{4}$ across the four slopes. $N_{2}$ is consistent with the change in $N_{3}$, thereby indicating that the goat performs a diagonal gait on the four slopes and that the change in foot pressure change is cyclical.

(3) The connection between joint angle and plantar pressure is: the greater the angle, the greater the pressure value. When the angle decreases to a certain value, the pressure value is zero, then the pressure value decreases again, and the pressure value is still zero.

Compared with the related previous works, imitation mountain-like walking institutions can adapt to the rugged ground, and the test data is more accurate. It was important to study the development of agricultural machinery and the development of agricultural bionic mechanism by accurately inputting the data obtained from this experiment into the walking mechanism. The measurement parameters provide the theoretical basis for the stable walking of the goat on the slope.

\section{Conclusions}

This scheme aimed to gain access to motion parameters to lay the foundation for future study of the stability of the four-legged walking mechanism and to provide conditions for its application. Four-legged bionic mechanism refers to the four-legged animal as a bionic prototype, all aspects of the optimal multi-coupling mechanism principle.

(1) As the speed increases, changes in the angle of the anterior and posterior limbs of the goat occur on the horizontal plane. The forelimbs support more weight than the hind limbs due to the static and physiological structures of the front and rear legs when the goat is in motion. The research will provide a theoretical basis for the design of agricultural walking mechanism to adapt different slopes in the hilly and mountainous areas.

(2) Gait is the main aspect of agricultural bionic institutions (using the analysis of four-legged animals, the bionic institutions are used in agriculture). Therefore, the goat walking test on different hillside slopes should be conducted before developing a agricultural bionic institution. Then the key parameters of gait on different slopes and the angle change curves of each goat leg in the process of slope movement were analyzed. The research will provide a theoretical basis for the design of agricultural walking mechanism to adapt different slopes in the hilly and mountainous areas.

\section{Acknowledgements}

The authors acknowledge that this research was financially supported by the National Natural Science Foundation of China (Grant No. 51675163), Scientific and Technological Project of Henan Province (Grant No. 152102210190, 162102210202), Foundation for University Young Key Teacher of Henan Province (Grant No. 2017GGJS062) and the postdoctoral fund of Henan Province (Grant No. 2012086).

\section{[References]}

[1] Chen D S, Zhang Z Q, Chen K W. Legs attitudes determination for bionic locust robot based on landing buffering performance. Mechanism and Machine Theory, 2016; 99: 117-139.

[2] Wang W, Wang $\mathrm{K}$, Zhang $\mathrm{H} \mathrm{X}$. Crawling gait realization of the mini-modular climbing caterpillar robot. Progress in Natural Science Materials International, 2009; 19(12): 1821-1829.

[3] Xuan Q P, Zhang H X, Dai G J. Stability gait planning of quadruped walking robot. Journal of Hangzhou Dianzi University, 2013; 33(2): 33-36. (in Chinese)

[4] Adrian M J, Roy W E, Karpovich P V. Normal gait of the dog: anele ctrogoniometric study. American Journal of Veterinary Research, 1966; 27: 90-95.

[5] Colin A, Lequang T, Maitre P, Viguier E. Limbs electrogoniometric study in a healthy trotting dog. Computer Methods in Biomechanics and Biomedical Engineering, 2008; 11(Supp 1): 61-62.

[6] Mantes J T. The dynamics of quadruped alwalking. Journal of Experimental Biology, 1938; 15: 522-540.

[7] Li H K, Dai Z D, Zhang H, Sun J R. Angular observation of joints of geckos moving on horizontal and vertical surfaces. Chinese Science Bulletin, 2009; 54(4): 592-598.

[8] Marghitu D B, Nalluri P. Nonlinear dynamic stability of normal and arthritic greyhounds. Nonlinear Dynamics, 1997; 12(3): 237-250.

[9] Roland E S, Hull M L, Stover S M. Design and demonstration of a dynamometric horseshoe for measuring ground reaction loads of horses during racing conditions. Journal of Biomechanics, 2005; 38(10): 2102-2112.

[10] Budsberg S C, Verstraete M C, Soutas-Little R W. Force plate analysis of the walking gait in healthy dogs. American Journal of Veterinary Research, 1987; 48(6): 915-918.

[11] Tashman S, Anderst W, Kolowich P, Havstad S, Arnoczky S. Kinematics of the ACL-deficient canine knee during gait: serial changes over two years. Journal of Orthopaedic Research, 2004; 22(5): 931-941.

[12] Tokuda K, Toda T, Koji Y, Konyo M, Tadokoro S, Alain P. Estimationof fragile ground by foot pressure sensor of legged robot. IEEE/ASMEInternational Conference on Advanced Intelligent Mechatronics, 2003; 1(1): 447-453.

[13] Piórek M, Josiński H, Michalczuk A, Świtoński A, Szczẹsna A. Quaternions and joint angles in an analysis of local stability of gait for different variants of walking speed and treadmill slope. Information Sciences, 2017; 384: 263-280.

[14] Liu C J, Wang D W, Goodman E D, Chen Q J. Adaptive walking control of biped robots using online trajectory generation method based on neural oscillators. Bionic Engineering Journal (English version), 2016; 13(4): $572-584$.

[15] Wang L W, Du W L, Wang X J, Xie G Z, Wang C D. A geometric approach to solving the stable workspace of quadruped bionic robot with hand-foot-integrated function. Robotics and Computer-Integrated Manufacturing, 2016; 37: 68-78.

[16] Deng H, Xin G Y, Zhong G L, Mistry M. Gait and trajectory rolling planning and control of hexapod robots for disaster rescue applications. Robotics \& Autonomous Systems, 2017; 95: 13-24.

[17] Di P, Hasegawa Y, Nakagawa S, Fukuda T, Huang J, Huang Q. Fall detection and prevention control using walking-aid cane robot. IEEE/ASME Transactions on Mechatronics, 2016; 21(2): 625-637.

[18] Capi G, Nasu Y, Barolli L, Mitobe K. Real time gait generation for autonomous humanoid robots: a case study for walking. Robotics \& 
Autonomous Systems, 2003; 42(2): 107-116.

[19] Chang J W, Wang R J, Chang C H, Chou H G, Wang W J. Fuzzy control-based real-time robust balance for a humanoid robot. Advanced Robotics, 2016; 30(19): 1-16.

[20] Huang J, Di P, Wakita K, Fukuda T. Study of fall detection using intelligent cane based on sensor fusion. International Symposium on Micro-nanomechatronics \& Human Science, 2008; pp.495-500.

[21] Agheli M, Nestinger S S. Force-based stability margin for multi-legged robots. Robotics \& Autonomous Systems, 2016; 83(c): 138-149.

[22] Agheli M, Nestinger S S. Foot force criterion for robot stability. International Conference on Climbing \& Walking Robots \& the Support Technologies for Mobile Machines, 2012; pp.417-424.

[23] Gu Z X, Zheng W C. Hole digging kinematic image acquisition of badger's forelimb digging hole. Journal of Economic Animal, 2013; 17(3): 152-154. (in Chinese)

[24] Meng Z X, Ma Y, Gu Z X, Su S F, Xu K H. Extraction method for the image of badger fore limb area based on HALCON. Journal of Economic Animal, 2012; 16(3): 159-162. (in Chinese)
[25] Gu Z X, Yao B, Xu K H, Liu Z S. Dynamic analysis on limbs of blue sheep and related technology. Development \& Innovation of Machinery \& Electrical Products, 2010; 23(5): 6-8. (in Chinese)

[26] Xu K H, Liu X L, Liu Z S, Gu Z X. The moving image process method of blue sheep based on Matlab. Development \& Innovation of Machinery \& Electrical Products, 2012; 25(1): 101-102. (in Chinese)

[27] Xu K H, Liu X L, Liu Z S, Gu Z X. Gait analysis of blue sheep based on identification technology. Journal of Economic Animal, 2011; 15(4): 193-197. (in Chinese)

[28] Song M J, Zhang M L. Kinematic analysis for parallel mechanism of bionic-mobile robot. Transactions of the CSAM, 2012; 43(3): 200-206. (in Chinese)

[29] Wang Y, Li J Q, Zhang G Q, Huang H, Zou M. Mechanical characteristics of bionic walking foot on soft sand. Transactions of the CSAM, 2016; 47(2): 384-389. (in Chinese)

[30] Zhang F, Wang W, Zhang G Y, Wang J, Qiu Z M. Gait analysis of goat at different slopes and study on biomimetic walking mechanism. Int J Agric \& Biol Eng, 2016; 9(3): 40-47. 